\title{
Neonatal citalopram exposure decreases serotonergic fiber density in the olfactory bulb of male but not female adult rats
}

\author{
Junlin Zhang ${ }^{1}$, Katie A. Dennis ${ }^{2}$, Ryan D. Darling ${ }^{1}$, Loai Alzghoul ${ }^{3}$, lan A. Paul ${ }^{4}$, \\ Kimberly L. Simpson ${ }^{1,4}$ and Rick C. S. Lin ${ }^{1,4 *}$ \\ ${ }^{1}$ Department of Neurobiology and Anatomical Sciences, The University of Mississippi Medical Center, Jackson, MS, USA \\ ${ }^{2}$ Neuroscience and Cognitive Studies, Millsaps College, Jackson, MS, USA \\ ${ }^{3}$ Program in Neuroscience, The University of Mississippi Medical Center, Jackson, MS, USA \\ ${ }^{4}$ Department of Psychiatry and Human Behavior, The University of Mississippi Medical Center, Jackson, MS, USA
}

\section{Edited by:}

Dirk Schubert, University Medical Centre Nijmegen, Netherlands

\section{Reviewed by:}

Barry Waterhouse, Drexel University College of Medicine, USA

Brent J. Thompson, University of

Texas Health Science Center,

San Antonio, USA

${ }^{*}$ Correspondence:

Rick C. S. Lin, Department of Neurobiology and Anatomical

Sciences, The University of

Mississippi Medical Center,

2500 North State Street,

Jackson, MS 39216, USA.

e-mail: rlin@umc.edu
Manipulation of serotonin $(5 \mathrm{HT})$ during early development has been shown to induce long-lasting morphological changes within the raphe nuclear complex and serotonergic circuitry throughout the brain. Recent studies have demonstrated altered raphe-derived $5 \mathrm{HT}$ transporter (SERT) immunoreactive axonal expression in several cortical target sites after brief perinatal exposure to selective $5 \mathrm{HT}$ reuptake inhibitors such as citalopram (CTM). Since the serotonergic raphe nuclear complex projects to the olfactory bulb (OB) and perinatal $5 \mathrm{HT}$ disruption has been shown to disrupt olfactory behaviors, the goal of this study was to further investigate such developmental effects in the OB of CTM exposed animals. Male and female rat pups were exposed to CTM from postnatal day 8-21. After animals reach adulthood (>90 days), OB tissue sections were processed immunohistochemically for SERT antiserum. Our data revealed that the density of the SERT immunoreactive fibers decreased $\sim 40 \%$ in the OB of CTM exposed male rats, but not female rats. Our findings support a broad and long-lasting change throughout most of the $5 \mathrm{HT}$ system, including the $\mathrm{OB}$, after early manipulation of $5 \mathrm{HT}$. Because dysfunction of the early $5 \mathrm{HT}$ system has been implicated in the etiology of neurodevelopmental disorders such as autism spectrum disorders (ASDs), these new findings may offer insight into the abnormal olfactory perception often noted in patients with ASD.

Keywords: serotonin transporter, olfactory bulb, selective serotonin reuptake inhibitors, sexual dimorphism, autism spectrum disorders

\section{INTRODUCTION}

Serotonin $(5 \mathrm{HT})$ is a widely distributed neuromodulator that plays an important role in regulating brain development (Gaspar et al., 2003). 5HT neurons in the dorsal raphe nucleus midline group and median raphe nucleus have axons distributed throughout the brain including the cortex (Waterhouse et al., 1986) and the olfactory bulb (OB) (McLean and Shipley, 1987). Manipulation of this system during early stages of neurodevelopment has been shown to produce sex-specific neurobehavioral modifications that persist well into adulthood (Csaba et al., 2003; Hohmann et al., 2007; Uçeyler et al., 2010). For example, serotonergic neurotoxin injections with 5,7-dihydroxytrypatmine into the bilateral medial forebrain bundles of neonatal mice induced long-term changes in social and sensory behaviors, as well as a depletion of the 5HT-immunoreactive (5HT-ir) fibers in the cerebral cortex (Boylan et al., 2007). Similarly, brief neonatal exposure to the selective serotonin reuptake inhibitor (SSRI) citalopram (CTM) lead to a sex-specific reduction of SERT-ir fibers in the neocortex $(\sim 40 \%)$ and hippocampus $(\sim 55 \%)$ of adult male rats (Maciag et al., 2006; Weaver et al., 2010). These male rats displayed reductions in novel object exploration, reduced social juvenile play (Rodriguez-Porcel et al., 2011), altered auditory sensory information processing, and reduced interhemispheric callosal connections (Simpson et al., 2011). In accordance with these findings seen in rats exposed neonatally to SSRIs, the inbred BTBR mice also display abnormalities in social juvenile play and have decreased $(\sim 20-30 \%)$ 5HT transporter (SERT) binding capacity throughout the brain (Gould et al., 2011). Depletion of $5 \mathrm{HT}$ in the $\mathrm{OB}$ affected olfactory recognition in neonate rats (Dulcy et al., 2010), and caused glomerular atrophy (Moriizumi et al., 1994). In addition, olfactory sensitivity has been shown to decrease after 3 weeks of CTM administration in adult mice (Lombion et al., 2008).

These lines of converging information demonstrate the importance of 5HT in the proper development of 5HT circuitry, and when manipulated via environmental and/or genetic means, lead to abnormal behaviors and altered innervation patterns within 5HT efferents. Therefore, our hypothesis was that brief neonatal CTM exposure will have a sex-specific effect on SERT-ir fibers within the $\mathrm{OB}$, with male abnormalities being more robust.

\section{MATERIALS AND METHODS}

All procedures were approved by the University of Mississippi Medical Center Institutional Animal Care and Use Committee 
and complied with the Association for Assessment and Accreditation of Laboratory Animal Care International and National Institutes of Health guidelines.

\section{ANIMALS AND TISSUE PREPARATION}

After the delivery of timed-pregnant Long Evans rats, the offspring were cross fostered to produce litters of $4 \sim 5$ pups. The pups were tattooed for identification on postnatal day (PN) 6, weaned at PN28, and housed in groups of $2 \sim 3 /$ cage under standard laboratory conditions with ad-libitum access to food and water. Beginning on PN8, the pups were injected subcutaneously with CTM (10 mg/kg, Tocris, Ellisville, MO) or saline in a volume of $0.1 \mathrm{ml}$ twice daily (total CTM dose of $20 \mathrm{mg} / \mathrm{kg} /$ day or saline volume of $0.2 \mathrm{ml} /$ day) for 14 days (PN8-21). This time window corresponds roughly from late stages of gestation to first 3 years of postnatal life in humans (Maciag et al., 2006). In addition, a non-treatment (NT) group (handled but without injection) was also included as a control for the effects of injection. The rationale behind the CTM dose was based on similar blood serum levels detected in rodents (Kugelberg et al., 2001) and humans (Bjerkenstedt et al., 1985), and similar dosages have been used and reported in previous rodent studies (Maciag et al., 2006; Weaver et al., 2010; Simpson et al., 2011).

After reaching adulthood $(\mathrm{PN}>60)$, animals were deeply anesthetized with pentobarbital $(75 \mathrm{mg} / \mathrm{kg}$, i.p.) and perfused through the ascending aorta with $0.9 \%$ saline, followed by $3.5 \%$ paraformaldehyde in $0.1 \mathrm{M}$ phosphate buffered saline (PBS). The brains were extracted and stored in 3.5\% paraformaldehyde solution and $25 \%$ sucrose overnight at $4^{\circ} \mathrm{C}$ until slicing.

\section{IMMUNOHISTOCHEMISTRY}

Rats from each treatment group were randomly divided into subsets and then processed and analyzed in these subsets to minimize immunostaining variability. For example, three rat brains (one non-treatment, one saline exposed, and one CTM exposed) were sliced and processed on the same day. Comparison of the SERT-ir fibers in rat $\mathrm{OB}$ was based initially on three male experimental subsets and three female experimental subsets. Two additional female sets were added to ensure no effect of treatment since no obvious alterations were noted in the female subsets.

In general, we followed the three-step indirect immunohistochemical procedures and quantification methods described previously (Maciag et al., 2006; Weaver et al., 2010). Specifically, $60 \mu \mathrm{m}$ coronal sections were taken throughout the OB using a freezing microtome and placed in individual wells. One out of every six sections were incubated in rabbit anti-SERT antibody (1:1000, Immunostar, Hudson, WI) for $24 \mathrm{~h}$ at room temperature followed by another $24 \mathrm{~h}$ at $4^{\circ} \mathrm{C}$. After rinse, sections were incubated in biotinylated anti-rabbit IgG (1:100, BA1000, ABC kit, Vector Laboratories, Burlingame, CA) for $1 \mathrm{~h}$ at room temperature. Axon profiles were then visualized by incubating the sections in Cy3-conjugated streptavidin (1:200, Jackson Immunoresearch Laboratories, Inc., West Grove, PA) for $1 \mathrm{~h}$ at room temperature in the dark. For each step of staining within a subset, total volumes of solution were created and distributed equally across animals to eliminate variability in concentrations within subsets. Finally, sections were mounted on gelatin-coated slides, allowed to air dry, and were then covered with DPX (mixture of distyrene, plasticizer, and xylene).

To control for non-specific labeling, two basic procedures were utilized. First, we conducted experiments where sections were processed according to the protocol, except that the primary SERT antiserum was omitted. Following this procedure, no SERT immunoreactivity was detected. Another control study also yielded negative immunostaining when an inappropriate secondary antibody such as biotinylated anti-mouse IgG (1:100, BA9200, ABC kit, Vector Laboratories, Burlingame, CA) was used for linkage.

\section{IMAGE ACQUISITION AND ANALYSIS}

Digital photomicrographs of sections containing the area of the main OB (Paxinos and Watson, 1986, bregma at $6.7 \mathrm{~mm}$ ) were taken with a consistent exposure time at $20 \times$ magnification using a Nikon E800 epifluorescent microscope equipped with a SenSys cool camera (Roper Scientific). The $20 \times$ magnification yielded $344 \times 437 \mu \mathrm{m}$ terminal field area. For each animal, three different sub-regions of the $\mathrm{OB}$ were chosen: the glomerular layer, the external plexiform layer, and the granule cell layer.

A previous study suggested that the glomerular layer in the dorsal and medial region of the OB has a higher number of SERTir fibers than those located in the lateral region (Gomez et al., 2005). Therefore, in order to reduce the sampling bias for the glomerular layer, we routinely took four images of the SERT-ir fibers from the middle portion of both medial and lateral regions of the OB. The same strategies of acquiring pictures were used for the external plexiform and the granule cell layers.

MetaMorph imaging software (Universal Imaging Systems) was used as described previously (Maciag et al., 2006; Weaver et al., 2010) to quantify the density of the SERT-ir fibers in the OB. Briefly, the signals on the photo were first adjusted to the same brightness. After thresholding the photo, the background was excluded and the SERT fibers were selected. A rectangular region $(125 \times 100 \mu \mathrm{m}$ for the glomerular layer, $437 \times 295 \mu \mathrm{m}$ for the external plexiform layer, and $437 \times 280 \mu \mathrm{m}$ for the granule cell layer) was created and placed on the photograph to include the specified layer. The area of this region also served as the reference point. The percentage of accumulated area of SERT fibers divided by the area of the rectangular region was defined as the SERT fiber density within this specific layer. The fiber density for each layer of an individual animal was determined by averaging the densities obtained from the four images. Data were analyzed statistically using MANOVAs with post-hoc Tukey's HSD tests (SPSS 19, IBM).

\section{RESULTS}

\section{DISTRIBUTION AND MORPHOLOGY OF SERT-ir FIBERS}

In both male and female control rats, the majority of SERT-ir fibers appear rather smooth and fine in caliber with very few varicosities (Figure 1A). Most of SERT-ir fibers in the granule cell layer tended to run parallel to the pia surface, and many of the SERT-ir fibers in the external plexiform layer were either perpendicular or parallel to the pia surface. In contrast, SERT-ir fibers in the glomerular layer were mostly randomly distributed. This 

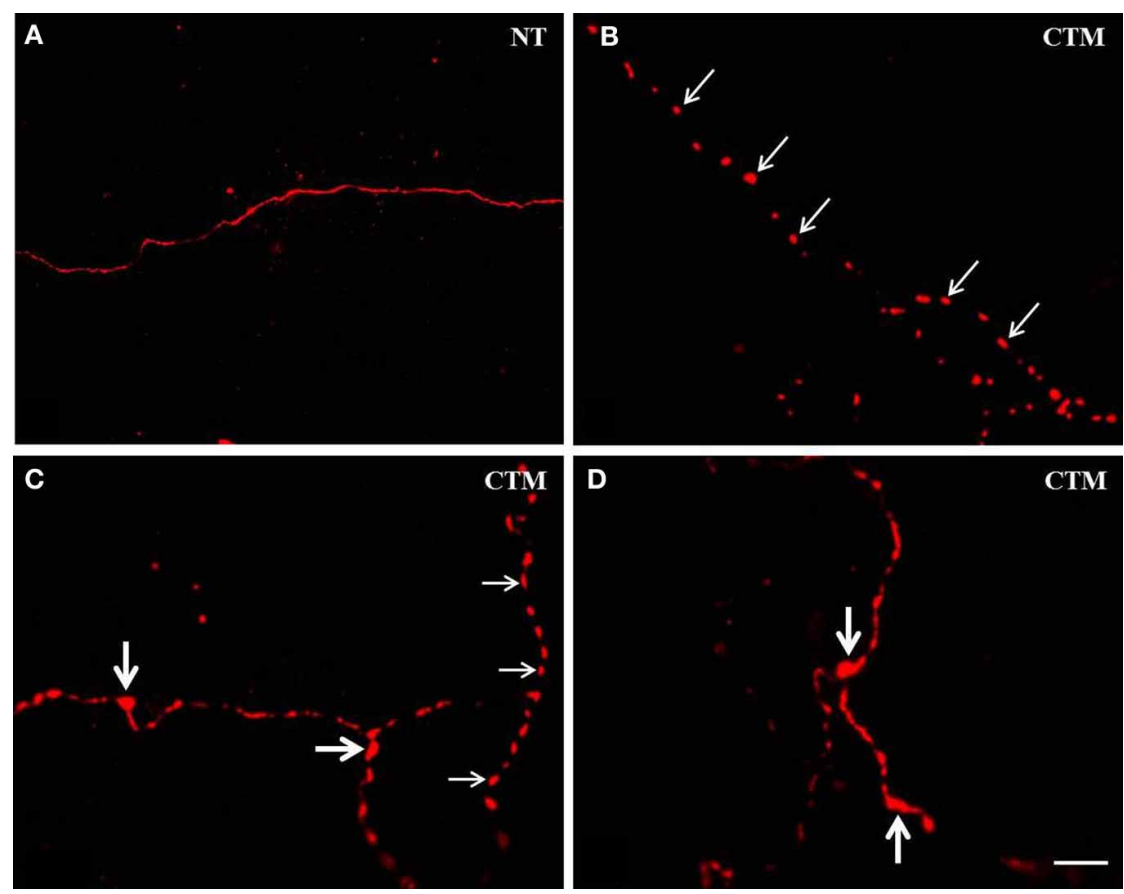

FIGURE 1 | Representative photomicrographs showing the altered morphology of SERT-ir fibers in the OB of male rats. (A) Normal SERT-ir fibers exhibit a smooth and fine morphology with very few varicosities. (B,C) Beaded-like SERT-ir fibers (thin arrows) were noted in

CTM exposed male rats. (C,D) The SERT-ir fibers with large bouton-like appearance (thick arrows) were noted in CTM exposed male rats. Scale bar $=2 \mu \mathrm{m}$. NT, non-treatment; CTM, citalopram; OB, olfactory bulb; SERT, serotonin transporter

general pattern of SERT-ir fiber distribution did not change in CTM exposed animals.

In contrast to the smooth and fine morphology of the SERT-ir fibers commonly seen in control animals, sex-specific varicosities were frequently noted in the glomerular layer of CTM exposed male animals (Figures 1B,C). These varicosities were found less frequently in the external plexiform and the granule cell layers. The distance between these varicosities along the SERT-ir fibers was usually very close and they were mainly in the range of $\sim 1-3 \mu \mathrm{m}$ apart. In addition, enlarged bouton-like swellings (Figures 1C,D) were occasionally noted in the granule cell layer of CTM exposed male rats. In contrast to these layer-specific morphological changes in male rats neonatally exposed to CTM, such changes were not seen in the female populations.

\section{DENSITY OF THE SERT-ir FIBERS IN MALE RATS}

Representative photos demonstrating the alteration of SERT-ir fiber density in the $\mathrm{OB}$ of male rats were shown in Figure 2. A one way MANOVA revealed a significant effect of treatment in the density of SERT-ir fibers within the glomerular layer of male rats, $F_{(2,6)}=10.82, p=0.010$, within the external plexiform layer, $F_{(2,6)}=6.47, p=0.032$, and within the granule layer, $F_{(2,6)}=$ $38.81, p<0.000$. Compared to SAL exposed rats, Tukey's HSD post-hoc tests revealed that SERT-ir fiber density within the OB of CTM exposed male rats was significantly reduced $\sim 39 \%$ in the glomerular layer $(p=0.015), \sim 38 \%$ in the external plexiform layer $(p=0.034)$, and $\sim 38 \%$ in the granule cell layer $(p=0.001)$ (Figure 3A). Importantly, there were no significant differences in
SERT-ir fiber density between NT and SAL exposed rats within any of the three OB layers ( $p \geq 0.79$ ) (Figure 3A), demonstrating that the effect was not due to the injections. The data from each experimental subset of male rats are listed in Table $\mathbf{1}$.

\section{DENSITY OF THE SERT-ir FIBERS IN FEMALE RATS}

Interestingly, SERT-ir fiber density within the OB of female rats showed a different pattern compared to that discovered in male rats. Representative photos were shown in Figure 4. Specifically, a one way MANOVA revealed a non-significant effect of treatment within the glomerular layer $F_{(2,12)}=0.173, p=0.843$, within the external plexiform layer $F_{(2,12)}=0.300, p=0.746$, and within the granule layer $F_{(2,12)}=0.464, p=0.640$ (Figure 3B). The data from each experimental subset of female rats are listed in Table 2.

\section{DISCUSSION}

We examined the neurodevelopmental effects of neonatal CTM exposure on the expression of SERT-ir fibers in the OB of adult rats. Our data revealed that disruption of the $5 \mathrm{HT}$ system during early life lead to a sex-specific and long-lasting change in the morphology and density of the SERT-ir fibers within the OB of adult male rats. This suggests a sexually dimorphic response to altered levels of neonatal 5HT, which is consistent with previous rodent studies (Csaba et al., 2003; Hohmann et al., 2007; Uçeyler et al., 2010), and further supports this model in the etiology of neurodevelopmental disorders (Rodriguez-Porcel et al., 2011; Simpson et al., 2011). 

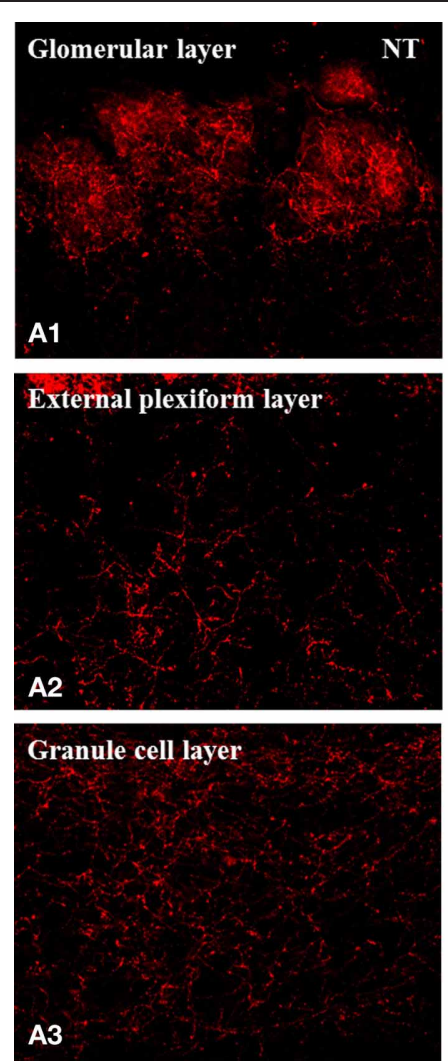
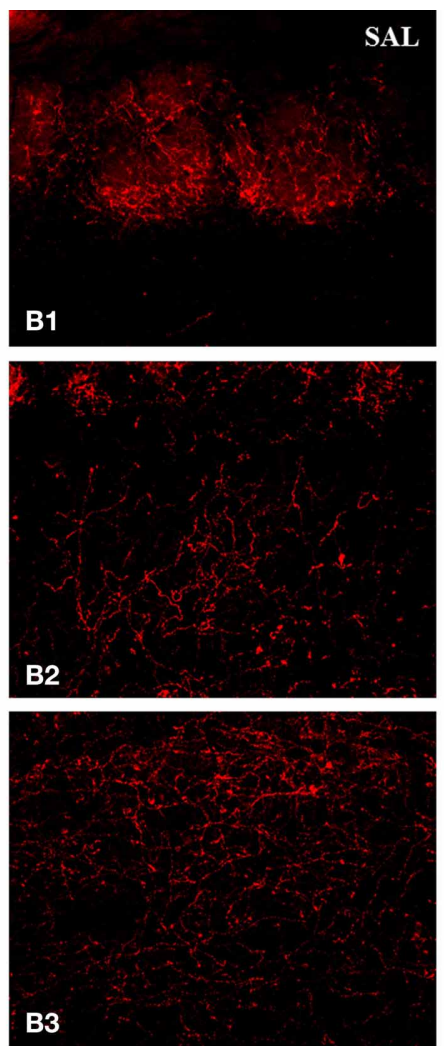
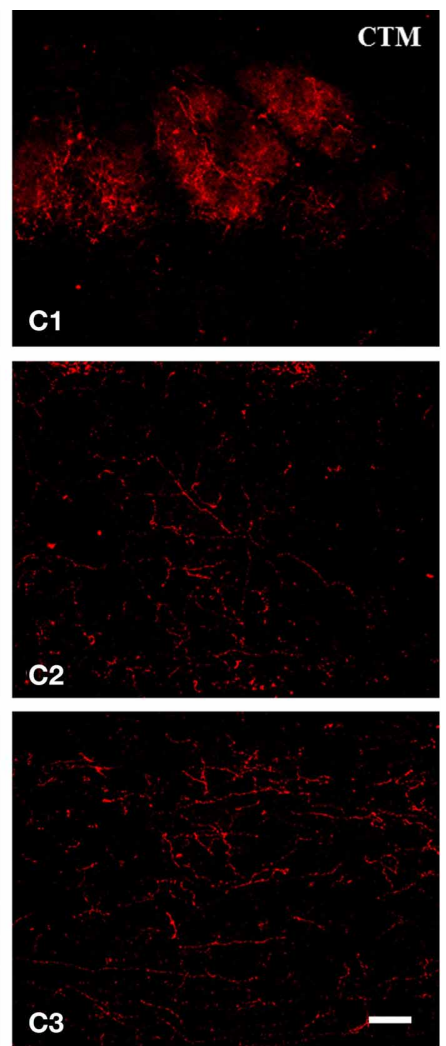

FIGURE 2 | Representative photomicrographs illustrating the distribution pattern of SERT-ir fibers in the $O B$ of male rats. (A1-C1) The glomerular layer. (A2-C2) The external plexiform layer. (A3-C3) The granule cell layer. Note that SERT-ir fiber density in CTM
(C1-C3) exposed rats was reduced in all three layers compared to NT (A1-A3) and SAL (B1-B3) exposed male rats. Scale bar $=50 \mu \mathrm{m}$. NT, non-treatment; SAL, saline; CTM, citalopram; OB, olfactory bulb; SERT, serotonin transporter.

\section{TECHNICAL CONSIDERATIONS}

It has been well-documented that the 5HT immunostaining patterns within the $\mathrm{OB}$ are reliably represented by using SERT immunostaining, but minor differences between the two biomarkers have been noted. For example, a greater number of SERT-ir fibers have been observed in the infraglomerular layer of the OB compared to 5HT-ir fibers, and the authors suggested that $5 \mathrm{HT}$ immunostaining may not effectively reveal neuronal processes in cells with a low concentration of intracellular 5HT (Gomez et al., 2005). Furthermore, the SERT protein was found within serotonergic axon bundles in addition to axon terminals, which makes it a useful biomarker for examining serotonergic axons (Zhou et al., 1998).

The layer-specific density of 5HT-ir fibers in the OB of normal adult male rats has been reported to be $\sim 8.8 \%$ in the glomerular layer, $\sim 3.5 \%$ in the granule cell layer, and $\sim 1.8 \%$ in the external plexiform layer (McLean and Shipley, 1987). In the current study, a similar pattern of the SERT-ir fiber density was observed in control animals with $\sim 13.3 \%$ in the glomerular layer, $\sim 8.4 \%$ in the granule cell layer, and $\sim 2.8 \%$ in the external plexiform layer, supporting the relative reliability of these two immunomarkers.

Appropriate measures were employed to ensure that our analysis was not biased by our staining and sampling procedures. For example, image quantification was done independently by two investigators blind to experimental conditions in order to verify interrater reliability. In addition, the staining procedures were processed in sets to minimize staining variability across groups. In fact, the Levene's test of equality of error variances was not significant within any of the three OB layers of male rats $(p \geq 0.604)$ or female rats $(p \geq 0.113)$, demonstrating equal error variances across groups.

\section{COMPARISON OF SERT-ir FIBER MORPHOLOGICAL CHANGES WITH OTHER STUDIES}

In the present study, varicosities $(\sim 1-3 \mu \mathrm{m}$ apart) on SERT-ir fibers were frequently noted in the glomerular layer of CTM exposed male rats, while these varicosities were less often found in the external plexiform and the granule cell layers. This is consistent with previous studies that found more 5HT-ir fibers with varicosities $(\sim 2-20 \mu \mathrm{m}$ apart $)$ in the glomerular layer compared to the infraglomerular layer (McLean and Shipley, 1987), and SERT-ir fibers with varicosities within the glomerular layer (Gomez et al., 2005) of normally developing rodents. The increased prevalence of dystrophic fibers seen in CTM exposed animals suggests altered function and may impair serotonergic signaling throughout the brain.

An increased number of dystrophic thick SERT-ir fibers has been noted in the amygdala, the hippocampus, and the cortex 


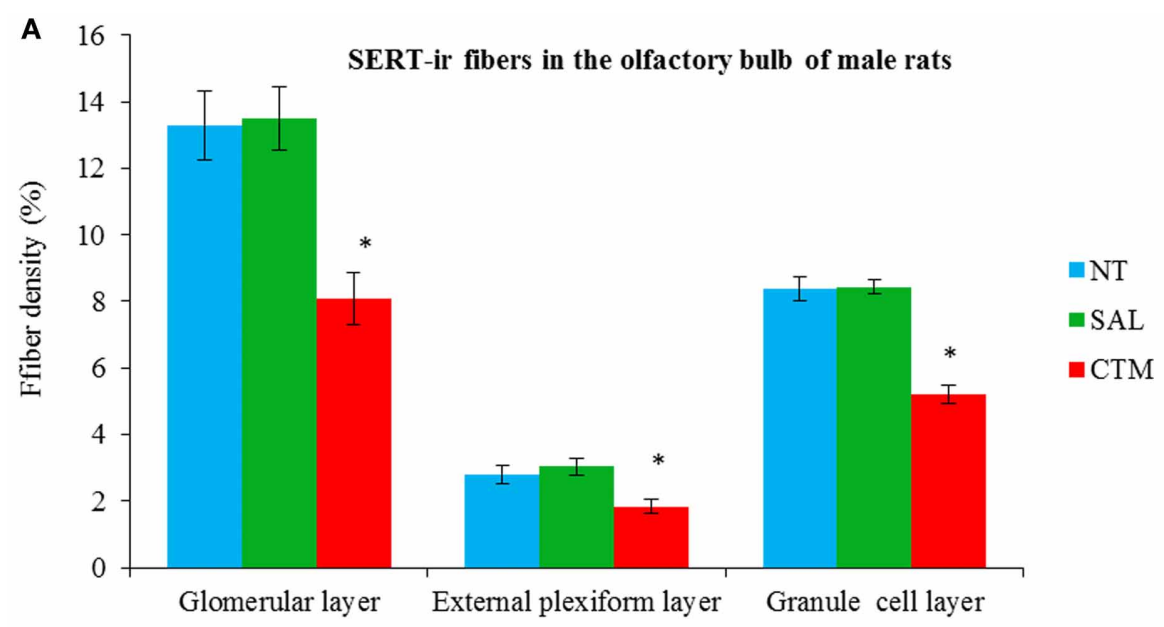

B

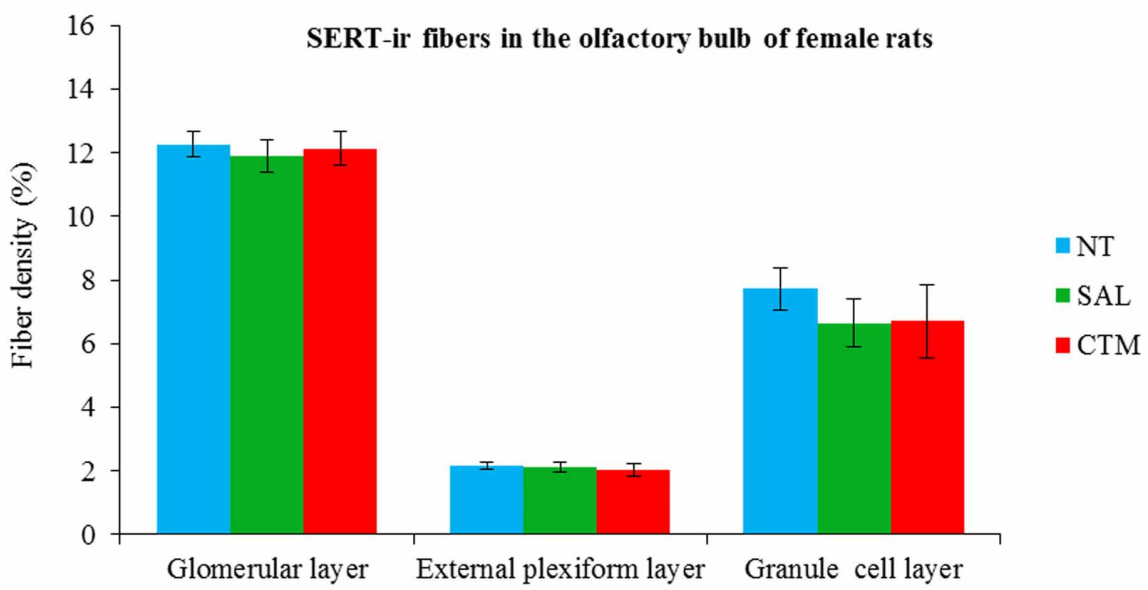

FIGURE 3 | Quantitative analysis of SERT-ir fiber density in the OB of both male and female rats. (A) The density of the SERT-ir fibers was significantly decreased in all three layers of the OB in CTM exposed male rats compared to SAL exposed male rats. (B) However, such changes were not significantly different in female rats. Error bars = SEM. *Represents $p<0.05$ compared to SAL group. NT, non-treatment; SAL, saline; CTM, citalopram; OB, olfactory bulb; SERT, serotonin transporter.

Table 1 | SERT-ir fiber density in the olfactory bulb of male rats.

\begin{tabular}{|c|c|c|c|c|c|c|c|c|c|}
\hline \multirow[t]{2}{*}{ Subset } & \multicolumn{3}{|c|}{ Glomerular layer (\%) } & \multicolumn{3}{|c|}{ External plexiform layer (\%) } & \multicolumn{3}{|c|}{ Granule cell layer (\%) } \\
\hline & NT & SAL & CTM & NT & SAL & CTM & NT & SAL & CTM \\
\hline 1 & 11.8 & 13.0 & 6.6 & 3.0 & 2.5 & 1.9 & 7.7 & 8.7 & 5.4 \\
\hline 2 & 15.3 & 15.3 & 8.4 & 3.1 & 3.3 & 2.1 & 8.5 & 8.0 & 5.6 \\
\hline 3 & 12.7 & 12.1 & 9.2 & 2.2 & 3.2 & 1.4 & 8.9 & 8.5 & 4.6 \\
\hline Mean & 13.3 & 13.5 & 8.1 & 2.8 & 3.0 & 1.8 & 8.4 & 8.4 & 5.2 \\
\hline SEM & 1.0 & 0.9 & 0.8 & 0.3 & 0.2 & 0.2 & 0.4 & 0.2 & 0.3 \\
\hline
\end{tabular}

NT, non-treatment; SAL, saline; CTM, citalopram; SEM, standard error of the mean.

of autistic patients (Azmitia et al., 2011). Interestingly, rodents exposed neonatally to CTM showed an increased number of thick and rod-like SERT-ir fibers and/or fine and beaded SERT-ir fibers in the cortex and hippocampus (Maciag et al., 2006; Weaver et al., 2010). However, in the present study, very few SERT-ir fibers in the OB showed this pattern after neonatal exposure to CTM. One possible explanation for this discrepancy could be that the neurochemical composition of the OB raphe projecting system may be different from cortical raphe projecting system. For example, the majority of cortical projecting $5 \mathrm{HT}$ neurons are in the midline 

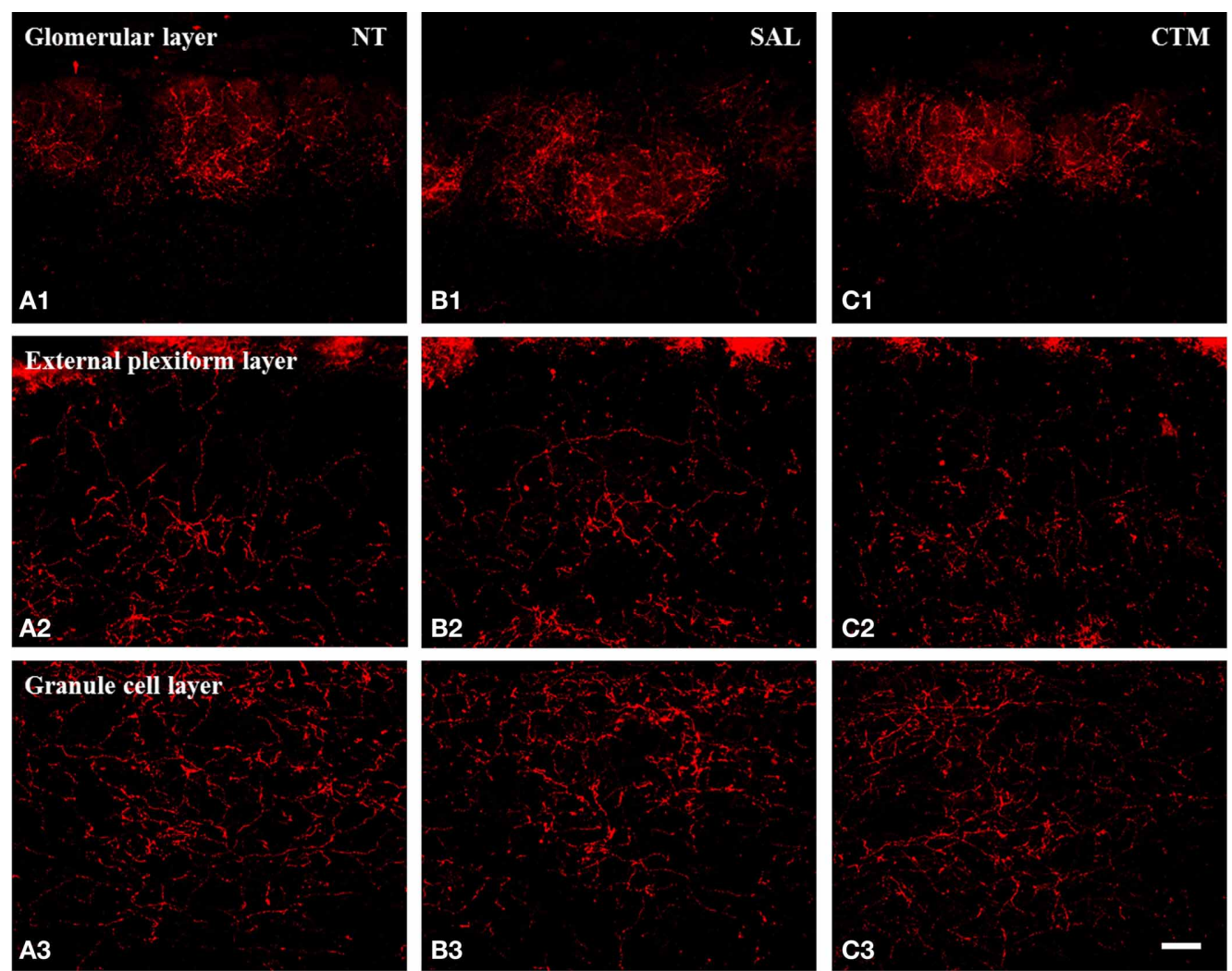

FIGURE 4 | Representative photomicrographs illustrating the distribution pattern of SERT-ir fibers in the OB of female rats. (A1-C1) The glomerular layer. (A2-C2) The external plexiform layer. (A3-C3) The granule cell layer. Note that SERT-ir fiber density remained unchanged in all three layers of NT (A1-A3), SAL (B1-B3), and CTM (C1-C3) exposed female rats. Scale bar $=50 \mu \mathrm{m}$. NT, non-treatment; SAL, saline; CTM, citalopram; OB, olfactory bulb; SERT, serotonin transporter.

Table 2 | SERT-ir fiber density in the olfactory bulb of female rats.

\begin{tabular}{|c|c|c|c|c|c|c|c|c|c|}
\hline \multirow[t]{2}{*}{ Subset } & \multicolumn{3}{|c|}{ Glomerular layer (\%) } & \multicolumn{3}{|c|}{ External plexiform layer (\%) } & \multicolumn{3}{|c|}{ Granule cell layer (\%) } \\
\hline & NT & SAL & CTM & NT & SAL & CTM & NT & SAL & CTM \\
\hline 1 & 11.9 & 11.8 & 13.6 & 1.9 & 2.6 & 2.6 & 8.8 & 5.3 & 10.4 \\
\hline 2 & 12.2 & 12.1 & 11.1 & 2.0 & 2.2 & 1.6 & 8.1 & 7.5 & 7.3 \\
\hline 3 & 12.1 & 10.0 & 11.2 & 2.2 & 1.8 & 1.6 & 6.1 & 7.3 & 6.2 \\
\hline 4 & 11.4 & 13.2 & 11.6 & 2.3 & 2.2 & 2.4 & 9.3 & 8.6 & 6.5 \\
\hline 5 & 13.8 & 12.3 & 13.2 & 2.5 & 1.7 & 1.8 & 6.3 & 4.6 & 3.1 \\
\hline Mean & 12.3 & 11.9 & 12.1 & 2.2 & 2.1 & 2.0 & 7.7 & 6.7 & 6.7 \\
\hline SEM & 0.4 & 0.5 & 0.5 & 0.1 & 0.2 & 0.2 & 0.7 & 0.7 & 1.2 \\
\hline
\end{tabular}

NT, non-treatment; SAL, saline; CTM, citalopram; SEM, standard error of the mean.

subgroup of the raphe complex co-express nitric oxide and 5HT (Simpson et al., 2003; Lu et al., 2010), and it is currently unknown whether this is the case for the $\mathrm{OB}$ projecting raphe neurons.

\section{SEXUAL DIMORPHISM OF THE 5HT SYSTEM}

At present, a limited number of studies have examined sexual differences within the developing 5HT system of human or rodent brains. It was reported that cortical 5HT content in normal male mice was twice that of female mice at PN3 (Connell et al., 2004).
In healthy young humans, $5 \mathrm{HT}$ synthesis capacity was higher in boys compared to girls (Chugani et al., 1999), and similarly higher in healthy adult men compared to women (Sakai et al., 2006). In contrast, 5HT levels were reported to be higher in the brainstem and limbic forebrain of female rats compared to male rats (Carlsson and Carlsson, 1988).

The major support for the sexually dimorphic development of the 5HT system is from rodent studies that have demonstrated sex-specific effects after early life manipulation of brain 
5HT levels. For example, it was reported that 5HT depleted male animals showed reduced exploration in response to spatial rearrangement and object novelty, however, this effect was not found in females (Hohmann et al., 2007). Similarly, physical activity was reduced in adult male SERT knock-out mice compared to females (Uçeyler et al., 2010). Furthermore, after a single injection of 5HT into newborn rats, 5HT levels were reduced in the striatum of adult male rats and displayed increased sexual activity, while no obvious differences were detected in adult female rats (Csaba et al., 2003). Interestingly, perinatal exposure to CTM affected male locus coeruleus circuit function but was not seen in females (Darling et al., 2011). Because a variety of early-life 5HT-system manipulations produce a constellation of long-term and sex-specific effects, it is not surprising that we observed a sexspecific reduction of SERT-ir fiber density in the OB of only male rats after neonatal CTM exposure.

Taken together, the current investigation and previous studies suggest that a sexual dimorphism does exist in both the normally developing 5HT system and after manipulations of brain 5HT levels. At present, the precise biological mechanism(s) of such sexual differences during early development are not known. Nonetheless, the neurodevelopmental consequences of these sexual differences need to be explored as it pertains to neurodevelopmental and psychiatric disorders that exhibit sex specificity.

\section{FUNCTIONAL SIGNIFICANCE AND CLINICAL IMPLICATIONS}

Recently, our laboratory and others have suggested that manipulation of perinatal 5HT levels induce numerous neurological and behavioral abnormalities, similar to what have been observed in autistic patients. For example, patients with autism spectrum disorder (ASDs) have been known to show impaired social interactions (Wing and Gould, 1979), a disrupted 5HT system in the brain (Chugani et al., 1999; Makkonen et al., 2008; Nakamura et al., 2010), as well as under-connectivity between two cortical hemispheres (Just et al., 2004; Vidal et al., 2006; Courchesne et al., 2007; Damarla et al., 2010). In addition, a recent study reported that exposure to SSRIs during pregnancy increased the risk of ASD diagnosis by a factor of more than two (Croen

\section{REFERENCES}

Azmitia, E. C., Singh, J. S., Hou, X. P., and Wiegel, J. (2011). Dystrophic serotonin axons in postmortem brains from young autism patients. Anat. Rec. 294, 1653-1662.

Bennetto, L., Kuschner, E. S., and Hyman, S. L. (2007). Olfactory and taste processing in autism. Biol. Psychiatry 62, 1015-1021.

Bjerkenstedt, L., Flyckt, L., Overo, K. F., and Lingjaerde, O. (1985). Relationship between clinical effects, serum drug concentration and serotonin uptake inhibition in depressed patients treated with citalopram. A double-blind comparison of three dose levels. Eur. J. Clin. Pharmacol. 28, 553-557.

Boylan, C. B., Blue, M. E., and Hohmann, C. F. (2007). Modeling

et al., 2011). These new lines of evidence suggest that dysregulation of 5HT levels may be one of the contributing factors for ASDs.

In addition to the core symptoms commonly seen in ASD, autistic patients are also known to display high incidence of sensory abnormalities (Rogers and Ozonoff, 2005). For example, it was reported that over $90 \%$ of children with ASDs had multiple sensory abnormalities (Leekam et al., 2007). Abnormal responses to multisensory information such as touch, oral, and olfactory stimuli have been proposed as predictors of the social severity in children with ASDs (Hilton et al., 2010). Other studies reported either disrupted olfactory identification (Suzuki et al., 2003; Bennetto et al., 2007; May et al., 2011) or altered odor sensitivity (Tomchek and Dunn, 2007; Dudova et al., 2011) in patients with ASDs. At present, the neurochemical and neuroanatomical foundations of the altered olfactory information processing in patients with ASDs remain unclear, but this model of earlylife 5HT manipulation may help elucidate the cause of these widespread sensory abnormalities.

Lastly, 5HT has been shown to play an important role in the rodent olfactory system, and disruption of 5HT levels lead to abnormal olfactory odor transmission. For example, 5HT was reported to be necessary in conditioned olfactory learning in rats (McLean et al., 1993; Moriizumi et al., 1994; Dulcy et al., 2010), mediated through $5 \mathrm{HT}_{2 \mathrm{~A} / 2 \mathrm{C}}$ receptors (McLean et al., 1996; Hardy et al., 2005). These previous findings along with our present data suggest a potential link between an altered 5HT system in the $\mathrm{OB}$ and the abnormal olfactory function noted in rats and patients with ASDs. However, it may be premature to suggest that an altered serotonergic system in the OB offers a possible clinical explanation for abnormal odor information process frequently noted in patients with ASDs. Therefore, additional behavioral tests of olfactory function in our animal model warrant further investigation.

\section{ACKNOWLEDGMENTS}

This work was supported by the National Institutes of Health under grant number: EUREKA MH084194.

A., Kennedy, D. P., et al. (2007). Mapping early brain development in autism. Neuron 56, 399-413.

Croen, L. A., Grether, J. K., Yoshida, C. K., Odouli, R., and Hendrick, V. (2011). Antidepressant use during pregnancy and childhood autism spectrum disorders. Arch. Gen. Psychiatry 68, 1104-1112.

Csaba, G., Knippel, B., Karabélyos, C. Inczefi-Gonda, A., Hantos, M., and Tekes, K. (2003). Impact of single neonatal serotonin treatment (hormonal imprinting) on the brain serotonin content and sexual behavior of adult rats. Life Sci. 73, 2703-2711.

Damarla, S. R., Keller, T. A., Kana, R. K., Cherkassky, V. L., Williams, D. L., Minshew, N. J., et al. (2010). Cortical underconnectivity coupled with preserved visuospatial cognition in autism: evidence from an fMRI study of an embedded figures task. Autism Res. 3, 1-7.

Darling, R. D., Alzghoul, L., Zhang, J., Khatri, N., Paul, I. A., Simpson, K. L., et al. (2011). Perinatal citalopram exposure selectively increases locus ceruleus circuit function in male rats. J. Neurosci. 31, 16709-16715.

Dudova, I., Vodicka, J., Havlovicova, M., Sedlacek, Z., Urbanek, T., and Hrdlicka, M. (2011). Odor detection threshold, but not odor identification, is impaired in children with autism. Eur. Child Adolesc. Psychiatry 20, 333-340.

Dulcy, C. P., Ganesh, A., and Rajan, E. (2010). Olfactory bulb serotonin level modulates olfactory 
recognition in the neonate rat. Indian J. Exp. Biol. 48, 1078-1082.

Gaspar, P., Cases, O., and Maroteaux, L. (2003). The developmental role of serotonin: news from mouse molecular genetics. Nat. Rev. Neurosci. 4, 1002-1012.

Gomez, C., Brinon, J. G., Barbado, M. V., Weruaga, E., Valero, J., and Alonso, J. R. (2005). Heterogeneous targeting of centrifugal inputs to the glomerular layer of the main olfactory bulb. J. Chem. Neuroanat. 29, 238-254.

Gould, G. G., Hensler, J. G., Burke, T. F., Benno, R. H., Onaivi, E. S., and Daws, L. C. (2011). Density and function of central serotonin (5HT) transporters, 5-HT1A and 5HT2A receptors, and effects of their targeting on BTBR $\mathrm{T}+\mathrm{tf} / \mathrm{J}$ mouse social behavior. J. Neurochem. 116, 291-303.

Hardy, A., Palouzier-Paulignan, B., Duchamp, A., Royet, J. P., and Duchamp-Viret, P. (2005). 5Hydroxytryptamine action in the rat olfactory bulb: in vitro electrophysiological path-clamp recordings of juxtaglomerular and mitral cells. Neuroscience 131, 717-731.

Hilton, C. L., Harper, J. D., Kueker, R. H., Lang, A. R., Abbacchi, A. M., Todorov, A., et al. (2010). Sensory responsiveness as a predictor of social severity in children with high functioning autism spectrum disorders. J. Autism Dev. Disord. 40, 937-945.

Hohmann, C. F., Walker, E. M., Boylan, C. B., and Blue, M. E. (2007). Neonatal serotonin depletion alters behavioral responses to spatial change and novelty. Brain Res. 1139, 163-177.

Just, M. A., Cherkassky, V. L., Keller, T. A., and Minshew, N. J. (2004). Cortical activation and synchronization during sentence comprehension in highfunctioning autism: evidence of underconnectitivy. Brain 127, 1811-1821.

Kugelberg, F. C., Apelqvist, G., Carlsson, B., Ahlner, J., and Bengtsson, F. (2001). In vivo steadystate pharmacokinetic outcome following clinical and toxic doses of racemic citalopram to rats. $\mathrm{Br}$. J. Pharmacol. 132, 1683-1690.

Leekam, S. R., Nieto, C., Libby, S. J., Wing, L., and Gould, J. (2007).
Describing the sensory abnormalities of children and adults with autism. J. Autism Dev. Disord. 37, 894-910.

Lombion, S., Morand-Villeneuve, N., and Millot, J. L. (2008). Effects of anti-depressants on olfactory sensitivity in mice. Prog. Neuropsychopharmacol. Biol. Psychiatry 32, 629-632.

Lu, Y., Simpson, K. L., Weaver, K. J., and Lin, R. C. (2010). Coexpression of serotonin and nitric oxide in the raphe complex: cortical versus subcortical circuit. Anat. Rec. 293, 1954-1965.

Maciag, D., Simpson, K. L., Coppinger, D., Lu, Y., Wang, Y., Lin, R. C., et al. (2006). Neonatal antidepressant exposure has lasting effects on behavior and serotonin circuitry. Neuropsychopharmacology 31, 47-57.

Makkonen, I., Riikonen, R., Kokki, H., Airaksinen, M. M., and Kuikka, J. T. (2008). Serotonin and dopamine transporter binding in children with autism determined by SPECT. Dev. Med. Child Neurol. 50, 593-597.

May, T., Brewer, W. J., Rinehart, N. J., Enticott, P. G., Brereton, A. V., and Tonge, B. J. (2011). Differential olfactory identification in children with autism and asperger's disorder: a comparative and longitudinal study. J. Autism Dev. Disord. 41, 837-847.

McLean, J. H., Darby-King, A., and Hodge, E. (1996). 5- $\mathrm{HT}_{2}$ receptor involvement in conditioned olfactory learning in the neonate rat pup. Behav. Neurosci. 110, 1426-1434.

McLean, J. H., Darby-King, A., Sullivan, R. M., and King, S. R. (1993). Serotonergic influence on olfactory learning in the neonate rat. Behav. Neural. Biol. 60, 152-162.

McLean, J. H., and Shipley, M. T. (1987). Serotonergic afferents to the rat olfactory bulb: I. origin and laminar specificity of serotonergic inputs in the adult rats. J. Neurosci. 7, 3016-3028.

Moriizumi, T., Tsukatani, T., Sakashita, H., and Miwa, T. (1994). Olfactory disturbance induced by deafferentation of serotonergic fibers in the olfactory bulb. Neuroscience 61, 733-738.

Nakamura, K., Sekine, Y., Ouchi, Y., Tsujii, M., Yoshikawa, E., Futatsubashi, M., et al. (2010). Brain serotonin and dopamine transporter bindings in adults with high-functioning autism. Arch. Gen. Psychiatry 67, 59-68.

Paxinos, G., and Watson, C. (1986). The Rat Brain in Stereotaxic Coordinates. Sydney, NSW: Academic press.

Rodriguez-Porcel, F., Green, D., Khatri, N., Harris, S. S., May, W. L., Lin, R. C. S., et al. (2011). Neonatal exposure of rats to antidepressants affects behavioral reactions to novelty and social interactions in a manner analogous to autistic spectrum disorders. Anat. Rec. 294, 1726-1735.

Rogers, S. J., and Ozonoff, S. (2005). Annotation: what do we know about sensory dysfunction in autism? A critical review of the empirical evidence. J. Child Psychol. Psychiatry 46, 1255-1268.

Sakai, Y., Nishikawa, M., Leyton, M. Benkelfat, C., Young, S. N., and Diksic, M. (2006). Cortical trapping of $\quad \alpha-\left[{ }^{11} \mathrm{C}\right]$ methyl-L-tryptophan, an index of serotonin synthesis, is lower in females than males. Neuroimage 33, 815-824.

Simpson, K. L., Waterhouse, B. D., and Lin, R. C. S. (2003). Differential expression of nitric oxide in serotonergic projection neurons: neurochemical identification of dorsal raphe inputs to rodent trigeminal somatosensory targets. J. Comp. Neurol. 466, 495-512.

Simpson, K. L., Weaver, K. J., de Villers-Sidani, E., Lu, J. Y., Cai, Z. Pang, Y., et al. (2011). Perinatal antidepressant exposure alters cortical network function in rodents. Proc. Natl. Acad. Sci. U.S.A. 108, 18465-18470.

Suzuki, Y., Critchley, H. D., Rowe, A., Howlin, P., and Murphy, D. G. M. (2003). Impaired olfactory identification in Asperger's syndrome. J. Neuropsychiatry Clin. Neurosci. 15, 105-107.

Tomchek, S. D., and Dunn, W. (2007). Sensory processing in children with and without autism: a comparative study using the short sensory profile. Am. J. Occup. Ther. 61, 190-200.

Uçeyler, N., Schütt, M., Palm, F., Vogel, C., Meier, M., Schmitt, A., et al. (2010). Lack of the serotonin transporter in mice reduces locomotor activity and leads to genderdependent late onset obesity. Int. J. Obes. 34, 701-711.

Vidal, C. N., Nicolson, R., DeVito, T. J., Hayashi, K. M., Geaga, J.
A., Drost, D. J., et al. (2006). Mapping corpus callosum deficits in autism: an index of aberrant cortical connectivity. Biol. Psychiatry 60, 218-225.

Waterhouse, B. D., Mihailoff, G. A., Baack, J. C., and Wodward, D. J. (1986). Topographical distribution of dorsal and median raphe neurons projecting to motor, sensorimotor, and visual cortical areas in the rat. J. Comp. Neurol. 249, 460-481.

Weaver, K. J., Paul, I. A., Lin, R. C., and Simpson, K. L. (2010). Neonatal exposure to citalopram selectively alters the expression of the serotonin transporter in the hippocampus: dose-dependent effects. Anat. Rec. 293, 1920-1932.

Wing, L., and Gould, J. (1979). Severe impairments of social interaction and associated abnormalities in children: epidemiology and classification. J. Autism Dev. Disorder 9, 11-29.

Zhou, F. C., Tao-Cheng, J. H., Segu, L., Patel, T., and Wang, Y. (1998). Serotonin transporters are located on the axons beyond the synaptic junctions: anatomical and functional evidence. Brain Res. 805, 241-254.

Conflict of Interest Statement: The authors declare that the research was conducted in the absence of any commercial or financial relationships that could be construed as a potential conflict of interest.

Received: 23 January 2013; accepted: 22 April 2013; published online: 10 May 2013.

Citation: Zhang J, Dennis KA, Darling RD, Alzghoul L, Paul IA, Simpson KL and Lin RCS (2013) Neonatal citalopram exposure decreases serotonergic fiber density in the olfactory bulb of male but not female adult rats. Front. Cell. Neurosci. 7:67. doi: 10.3389/fncel. 2013.00067

Copyright (c) 2013 Zhang, Dennis, Darling, Alzghoul, Paul, Simpson and Lin. This is an open-access article distributed under the terms of the Creative Commons Attribution License, which permits use, distribution and reproduction in other forums, provided the original authors and source are credited and subject to any copyright notices concerning any third-party graphics etc. 